\title{
Recent Advances in Computed Tomography Imaging in Chronic Obstructive Pulmonary Disease
}

\author{
Sandeep Bodduluri ${ }^{1,2}$, Joseph M. Reinhardt ${ }^{3}$, Eric A. Hoffman ${ }^{3}$, John D. Newell Jr. ${ }^{3}$, and Surya P. Bhatt ${ }^{1,2,4}$ \\ ${ }^{1}$ Division of Pulmonary, Allergy, and Critical Care Medicine, and ${ }^{2}$ University of Alabama at Birmingham Lung Imaging Core, University of \\ Alabama at Birmingham, Birmingham, Alabama; ${ }^{3}$ Department of Radiology and Biomedical Engineering, University of lowa, lowa City, \\ lowa; and ${ }^{4}$ Lung Health Center, University of Alabama at Birmingham, Birmingham, Alabama
}

\begin{abstract}
Lung imaging is increasingly being used to diagnose, quantify, and phenotype chronic obstructive pulmonary disease (COPD).

Although spirometry is the gold standard for the diagnosis of COPD and for severity staging, the role of computed tomography (CT) imaging has expanded in both clinical practice and research. COPD is a heterogeneous disease with considerable variability in clinical features, radiographic disease, progression, and outcomes. Recent
\end{abstract}

studies have examined the utility of CT imaging in enhancing diagnostic certainty, improving phenotyping, predicting disease progression and prognostication, selecting patients for intervention, and also in furthering our understanding of the complex pathophysiology of this disease. Multiple CT metrics show promise for use as imaging biomarkers in COPD.

Keywords: computed tomography; imaging; image registration; emphysema; small airways

(Received in original form May 8, 2017; accepted in final form July 24, 2017)

Supported by National Institutes of Health grant K23HL133438 (S.P.B.).

Author Contributions: S.B. and S.P.B. contributed to the drafting of the manuscript. S.B., J.M.R., E.A.H., J.D.N., and S.P.B. assisted with the critical revision of the manuscript for important intellectual content.

Correspondence and requests for reprints should be addressed to Surya P. Bhatt, M.D., University of Alabama at Birmingham, Division of Pulmonary, Allergy, and Critical Care Medicine, THT 422, 1720, 2nd Avenue South, Birmingham, AL 35294. E-mail: sbhatt@uabmc.edu.

Ann Am Thorac Soc Vol 15, No 3, pp 281-289, Mar 2018

Copyright (C) 2018 by the American Thoracic Society

DOI: 10.1513/AnnalsATS.201705-377FR

Internet address: www.atsjournals.org

The diagnosis of chronic obstructive pulmonary disease (COPD) has traditionally relied on spirometry. This reliance on spirometry for diagnosis and severity assessment has led to modest advances in our understanding of underlying pathophysiology. COPD is increasingly recognized as a complex heterogeneous disease, and recent advances in computed tomography (CT) have enabled extensive phenotyping of COPD by allowing morphologic characterization of parenchymal and airways disease $(1,2)$. CT enables visualization of structural derangements and hence anatomic localization of disease, in contrast to spirometry, which is a more global measure. Indeed, substantial disagreement can exist between spirometric assessment of airflow obstruction and quantitation of structural lung disease (3). Visual analysis can provide semiquantitative estimates of overall degree of emphysema, as well as of emphysema subtypes (4). In combination with qualitative estimates of airway disease, visual analysis has enabled phenotyping of COPD into emphysema and airways-predominant disease, with implications for respiratory morbidity and disease outcomes (5).

Automated density mask analyses have facilitated quantitation of lowattenuation areas on inspiratory scans to measure the percentage of lung parenchyma affected by emphysema (4). Using differential thresholds for lung density can provide estimates for mild and severe emphysema (4). Airway segmentation and measurement of segmental and subsegmental airway wall thickness allow quantification of airways disease, although these measurements are limited to the first few generations of airway branching, due to limitations in CT resolution. Details of these advances in CT image-based quantification and assessment of COPD have been discussed elsewhere $(1,2,6-8)$.

Recently, several large, multicenter studies combined information from both inspiratory and expiratory CT scans to extract clinically relevant measures of COPD. Inspiratory scans are obtained at total lung capacity (TLC) and expiratory scans at functional residual capacity (FRC) or residual volume. Expiratory scans allow quantification of gas trapping, an indirect measure of small airway disease that cannot be directly visualized with existing CT resolution. Inspiratory and expiratory images can be matched voxel to voxel, and assessments of regional lung expansion can be made. Using image post-processing tools, 
paired CT scans obtained at two different lung volumes allow a rich and diverse range of measurements that substantially add to our understanding of disease pathophysiology. These techniques have highlighted the heterogeneity of disease processes in COPD, especially in early disease stages, where intervention may be more fruitful. In this Focused Review, we provide an overview of recent advances in using combined inspiratory and expiratory CT images for assessment of parenchymal and airways disease in COPD. We summarize the strong correlations of the new metrics with existing spirometric measures for disease diagnosis and severity assessment, and portray the value of these new measures by showing that these novel metrics explain respiratory morbidity and outcomes, independent of spirometry measures.

\section{Computed Tomography Densitometry}

\section{Emphysema}

Visual estimates of emphysema are semiquantitative and rely on estimates of lung density as well as visual patterns (4). Emphysematous lung can also be objectively quantified by commercially available computer software using selected thresholds of tissue attenuation measurement (Hounsfield units [HU]) on inspiratory images obtained at TLC. The number of voxels below this threshold expressed as a fraction of the total number of voxels provides a measure of emphysema. Thresholds of $-910 \mathrm{HU}$ and $-950 \mathrm{HU}$ have been used to quantify mild and severe emphysema, respectively (9-11), and both thresholds have been validated with histopathology (12-15). Multiple clinical studies have adapted these thresholds, providing data on patient selection for interventional studies (16), gene associations (17), and associations with respiratory morbidity (5).

\section{Small Airway Disease}

The small conducting airways less than $2 \mathrm{~mm}$ in internal diameter are the major site of airflow obstruction in COPD, but these cannot be directly visualized on CT. Density mask analyses of expiratory scans can quantify gas trapping, which reflects increased air, and hence lower lung density at end expiration, resulting from a combination of decreased elastic recoil due to emphysema and increased small airway resistance. Densitometric results are substantially different in chronic bronchitis compared with controls on CT scans obtained at $90 \%$ and $10 \%$ of vital capacity, respectively (18). The most accepted threshold for gas trapping, assessed on expiratory scans, is $-856 \mathrm{HU}$, although other thresholds have also been used (19). Gas trapping quantified using the $-856 \mathrm{HU}$ threshold correlates strongly with spirometric airflow obstruction $(r=-0.82)(20)$.

A major limitation of the $-856 \mathrm{HU}$ fixed-density threshold on expiration is the inability to distinguish low-density regions due to emphysematous tissue destruction from gas trapping in more normalappearing lung regions resulting from small airways disease. A number of investigators have attempted to account for the emphysema part and derive more specific measures of small airways disease $(20,21)$. Matsuoka and colleagues (21) excluded all voxels less than $-950 \mathrm{HU}$ as emphysematous voxels, and then calculated the percent change of relative area, with attenuation values from -860 to $-950 \mathrm{HU}$ between inspiratory and expiratory CT scans. They showed that this relative volume change strongly correlated with $\mathrm{FEV}_{1} / \mathrm{FVC}(-0.78)$ and $\mathrm{FEV}_{1} \%$ predicted $(-0.80)(21)$. Hersh and associates (20) studied an alternative measure of gas trapping adjusted for emphysema by calculating the ratio of the expiratory to inspiratory mean lung density (E/I). The $\mathrm{E} / \mathrm{I}$ ratio correlated with both $\mathrm{FEV}_{1} / \mathrm{FVC}$ $(r=-0.62)$ and $\mathrm{FEV}_{1} \%$ predicted $(r=-0.73)$, as well as with patient outcomes, such as dyspnea, respiratory quality of life, and 6-minute walk distance. They found that, especially in subjects with more severe emphysema, the E/I ratio, as well as the relative volume change from $-856 \mathrm{HU}$ to $-950 \mathrm{HU}$, yielded more predictive associations with outcomes than measures using fixed thresholds, suggesting that these metrics provided additional information on small airway disease by adjusting for emphysema measures. Although these represent advances in assessing small airway disease, these measures provide adjustments for the global presence of emphysema, and lack the anatomic localization to truly adjust for emphysema by region.

\section{Large Airway Disease}

In contrast to small airways, large airways above the sixth generation can be easily visualized on both inspiratory and expiratory scans. Segmental and subsegmental airway wall thickness has been used to quantify airway disease, with the assumption that changes at this level reflect more distal changes in the airway tree. Common measurements include airway wall thickness, airway wall area percent $(\mathrm{WA} \%=$ [outer area of airway - lumen area] / outer area of airway $\times 100$ ), and square roots of the wall areas of hypothetical airways with internal perimeters (Pis) of 10 and $15 \mathrm{~mm}$ (Pi10 and Pi15, respectively) (22-25). The Pi10 and Pi15 measurements were devised to avoid bias due to betweensubject differences in airway sizes. The square root of the airway wall thickness was plotted against the Pi of each airway, and the regression line was used to derive the square root of the wall area for a "hypothetical airway" with Pi10 or Pi15. These metrics correlate with $\operatorname{FEV}_{1}(26,27)$, bronchodilator responsiveness (28), paradoxical response to bronchodilators (29), and respiratory morbidity independent of emphysema (30), and also are associated with subsequent lung function decline (26). Pi10 has been reported to be greater in patients without evident airflow obstruction, but with respiratory symptoms (31), and a greater Pi10 can predict the development of airflow obstruction on longitudinal follow-up (26). Wall area thickness expressed as a percentage of overall WA\% increases with increasing disease severity, but, at each airway generation, luminal diameter decreases disproportionately with worsening disease compared with wall area itself, and recent studies show progressively thinner airway walls with worsening disease severity $(32,33)$. Washko and colleagues (34) assessed the density of airway walls and showed that wall attenuation is associated with lung function, even after adjustment for emphysema and WA\%, suggesting that this measure offers additional information on the characteristics of the larger airways.

In addition to changes in wall thickness, airway walls are also frequently 
less rigid and more collapsible in patients with COPD, and the degree of airways collapse on expiration can be assessed on expiratory CT. Expiratory central airway collapse (ECAC) includes expiratory dynamic airway collapse due to weak posterior membrane of the larger airways, as well as tracheomalacia due to cartilaginous weakness. Although the small conducting airways offer the most resistance to airflow in COPD, collapse greater than $50 \%$ of the larger central airways during exhalation likely causes additional airflow obstruction. Traditionally diagnosed on bronchoscopy or using dynamic CT during exhalation, ECAC visualized on dualvolume static CT is common in COPD, and is present in about $5 \%$ of patients with COPD (35). In a large study of 8,820 current and former smokers, Bhatt and colleagues (35) showed that the presence of
ECAC is associated with worse respiratory quality of life, greater dyspnea, and a higher frequency of exacerbations.

\section{Image Matching}

To improve anatomic localization and enhance the separate assessment of emphysema and small airway disease, spatial alignment of inspiratory and expiratory images is necessary. This can be achieved by image matching, also referred to as image registration, which performs the task of spatial alignment or voxel-by-voxel mapping, either between two-dimensional images or three-dimensional volumes. Image matching is usually performed between inspiratory and expiratory CT scans, such that one image is spatially deformed to match the other using defined anatomic landmarks, such as airways (Figure 1). The procedure can also be performed between baseline and follow-up scans in longitudinal studies to track regional disease progression. Multiple algorithms for image registration exist, the technical details of which have been described previously (36). There are two fundamental approaches to extract clinically relevant information from the image registration process (Figure 1). The first approach is to perform a voxel-byvoxel anatomic comparison and assess the corresponding CT density change from expiration to inspiration, with compensation for lung deformation through image registration. The second approach is to use the lung deformation between inspiration and expiration to derive mechanical and functional measures of lung parenchyma.
Inspiration CT

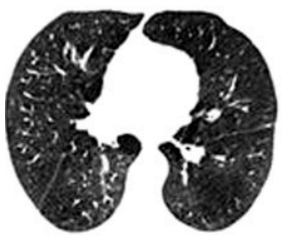

Expiration CT

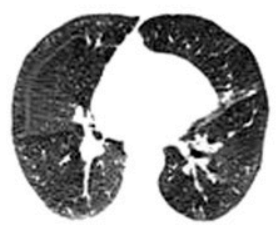

Structural Measure

Classifying voxels into emphysema and non-emphysematous functional small airway disease using voxel-by-voxel comparison between deformed inspiration and expiration CT images
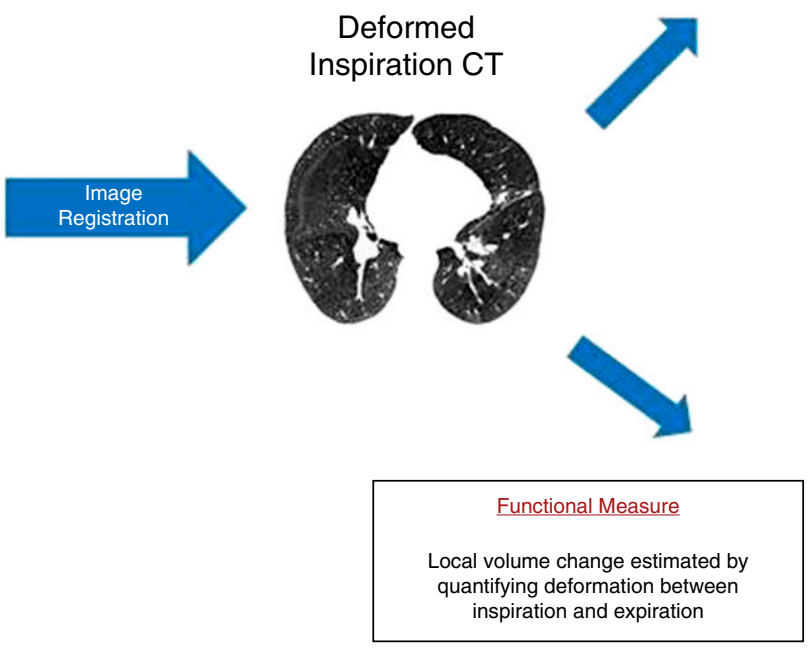

\section{Parametric Response} Mapping (PRM)

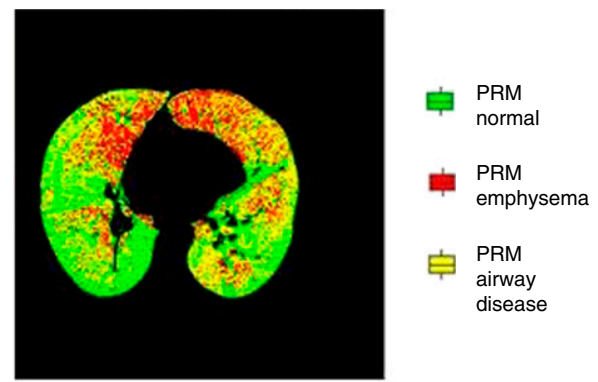

Local Volume Change (Jacobian Determinant)

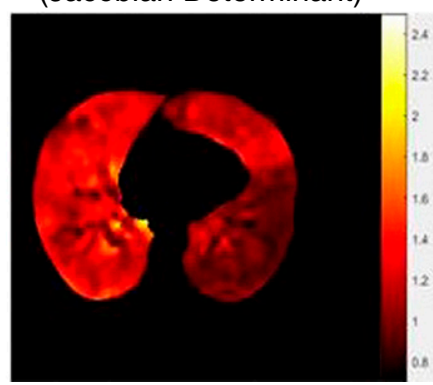

Figure 1. The technique of image registration and two fundamental approaches to extracting clinically relevant information from image registration. Inspiratory and expiratory images are matched voxel-by-voxel. Structural measures can be obtained by performing a voxel-by-voxel anatomic comparison and assessing the corresponding computed tomography (CT) density change from expiration to inspiration, with compensation for lung deformation through image registration. The top right shows a representative axial section with localization of emphysema (red), functional small airways disease (yellow), and normal (green) voxels in a patient with moderate chronic obstructive pulmonary disease (COPD). The bottom right depicts functional changes on the same slice. Here, the amount of lung deformation between inspiration and expiration is used to derive a measure of regional ventilation, termed the Jacobian determinant, a measure of local volume change from full inspiration to end expiration. The Jacobian determinant ranges from 0 to infinity; values greater than 1 indicate local expansion, and values less than 1 indicate local contraction. PRM= parametric response mapping. 


\section{Structural Lung Disease}

\section{Small Airway Disease}

Parametric response mapping (PRM) is a recently introduced application of image matching, whereby inspiratory and expiratory images are matched on a voxel-by-voxel basis to examine the differences in density between inspiratory and expiratory images (37). For all voxel pairs within the registered inspirationexpiration lungs, PRM classifies individual voxel pairs based on the commonly used fixed CT density thresholds that represent emphysema ( $-950 \mathrm{HU}$ on inspiratory CT) and gas trapping $(-856$ HU on expiratory CT). Voxels with density less than $-950 \mathrm{HU}$ on inspiratory CT and less than $-856 \mathrm{HU}$ on expiratory CT are deemed emphysematous voxels $\left(\mathrm{PRM}^{\mathrm{Emph}}\right)$, and voxels with density greater than $-950 \mathrm{HU}$ on inspiratory CT and less than $-856 \mathrm{HU}$ on expiratory CT scan represent areas of "pure" gas trapping without contribution from emphysema, and thus a measure of functional small airways disease $\left(\mathrm{PRM}^{\mathrm{fSAD}}\right)$. The term "functional" is used, as this metric does not directly visualize small airway disease, but instead provides a more homogeneous measure of nonemphysematous air trapping.

As seen with earlier density thresholdbased gas trapping metrics, PRM measures correlate strongly with lung function, and $\mathrm{PRM}^{\mathrm{fSAD}}$ is inversely related to $\mathrm{FEV}_{1} / \mathrm{FVC}$ $\left(r^{2}=0.545\right)$ and to $\mathrm{FEV}_{1}\left(r^{2}=0.517\right)$, independent of emphysema (37). In mild to moderate COPD (GOLD [Global Initiative for Chronic Obstructive Lung Disease] stages 1-2), density-based CT gas trapping and $\mathrm{PRM}^{\mathrm{fSAD}}$ show similar correlations with $\mathrm{FEV}_{1}$; however, the two measures show differential trends in higher disease stages (GOLD stages 3-4), as increasing emphysema adds to the gas trapping fraction (37). It should be noted that density-based CT emphysema and $\mathrm{PRM}^{\mathrm{Emph}}$ provide identical measures of emphysema, as they do not account for expiratory changes.

PRM provides spatial information on disease location and subtype, and PRM metrics can serve as imaging biomarkers for disease progression. Studies using $\mathrm{PRM}^{\mathrm{fSAD}}$ as a measure of small airway disease appear to corroborate micro-CT data, suggesting that small airways disease precedes emphysema development in COPD (37,
38). In a large study of 1,508 current and former smokers with and without COPD, Bhatt and associates (39) showed that both $\mathrm{PRM}^{\mathrm{fSAD}}$ and PRM ${ }^{\text {Emph }}$ were associated with $\mathrm{FEV}_{1}$ decline in patients with COPD; however, in those at risk, but without overt airflow obstruction, $\mathrm{PRM}^{\mathrm{fSAD}}$, but not $\mathrm{PRM}^{\text {Emph }}$, was associated with $\mathrm{FEV}_{1}$ decline. Using sequential, paired CT scans at baseline, 30 days, and 12 months, Boes and colleagues (40) showed that PRM metrics are reproducible. They evaluated temporal changes in emphysema and small airways disease across a range of disease severity over a 12-month period and found that there is an early increase in $\mathrm{PRM}^{\mathrm{fSAD}}$ in mild disease followed by a decrease in PRM ${ }^{\mathrm{fSAD}}$ even as PRM ${ }^{\mathrm{Emph}}$ increases with increasing disease severity. Some of the PRM ${ }^{\mathrm{fSAD}}$ appears to be reversible, and may represent airway inflammation, mucus plugging, or bronchospasm as opposed to airway fibrosis or disappearance (40). The anatomical localization provided by image registration can prove useful to track disease progression locally. Correlations of these metrics with histopathology are currently underway.

\section{Emphysema}

Lung density measurement remains the best available metric to track structural disease progression in clinical studies (41), and this has been used to track response to augmentation therapy in patients with $\alpha_{1}$-antitrypsin deficiency $(42,43)$. A major challenge in the assessment of disease progression on $\mathrm{CT}$ is variability in the lung volume at which serial CT scans are acquired, as changes in TLC can affect lung density measurements. Measurements of disease progression are also influenced by scanner variability. Change in smoking status between scans can also affect lung density, with active smoking resulting in higher lung density than not smoking (44). Efforts are being made to standardize lung volumes during CT acquisition, and phantoms are being used to ameliorate scanner variability (25). A further limitation of these density measures is that they are global assessments of disease, with incremental changes over time. Image registration can be employed to match inspiratory scans obtained serially to assess emphysema progression $(45,46)$. Gorbunova and colleagues (47) used image registration in a small study of 27 patients with severe emphysema to match serially acquired images at baseline, and at 3, 12, 21, and 24-30 months, and found that measurement of local disease progression could be consistently demonstrated. Image registration-based algorithms have been proposed for the measurement of local progression of emphysema that is minimally affected by changes in lung volume between baseline and follow-up $(48,49)$.

\section{Functional Measures}

The heterogeneity of disease initiation and progression implies that there is no uniform trajectory from an absence of disease to the development of airflow obstruction, and that there are trajectories in disease progression beyond that measured by $\mathrm{FEV}_{1}$ alone. $\mathrm{FEV}_{1}$ alone is likely not sufficient to measure disease progression. In addition to structural changes in the lung that precede the development of airflow obstruction, there are likely local functional changes that may initiate or aid progression of disease (50), changes that can be assessed by CT image registration at a regional level. Functional measures based on the deformation of the lung during respiration may enhance our understanding of the pathophysiology of $\mathrm{COPD}$, and may result in more sensitive measures for detection of emphysema (51). Two classes of registration-based measures of regional function of lung parenchyma can be derived: regional ventilation measures (local volume change) and measures of lung tissue mechanics (Figure 2).

\section{Regional Ventilation}

Density difference. Spirometry provides a global measure of volume change from inspiration to expiration. It is increasingly apparent that a substantial amount of functional loss at the regional level can exist before detection of disease by spirometry $(31,52)$. Given that lung tissue remains constant during the respiratory cycle, and disregarding vascular volume changes, there is a normal increase in CT attenuation from end-inspiration to endexpiration, and the change in lung density from inspiration to expiration reflects change in air volume. Air trapping 


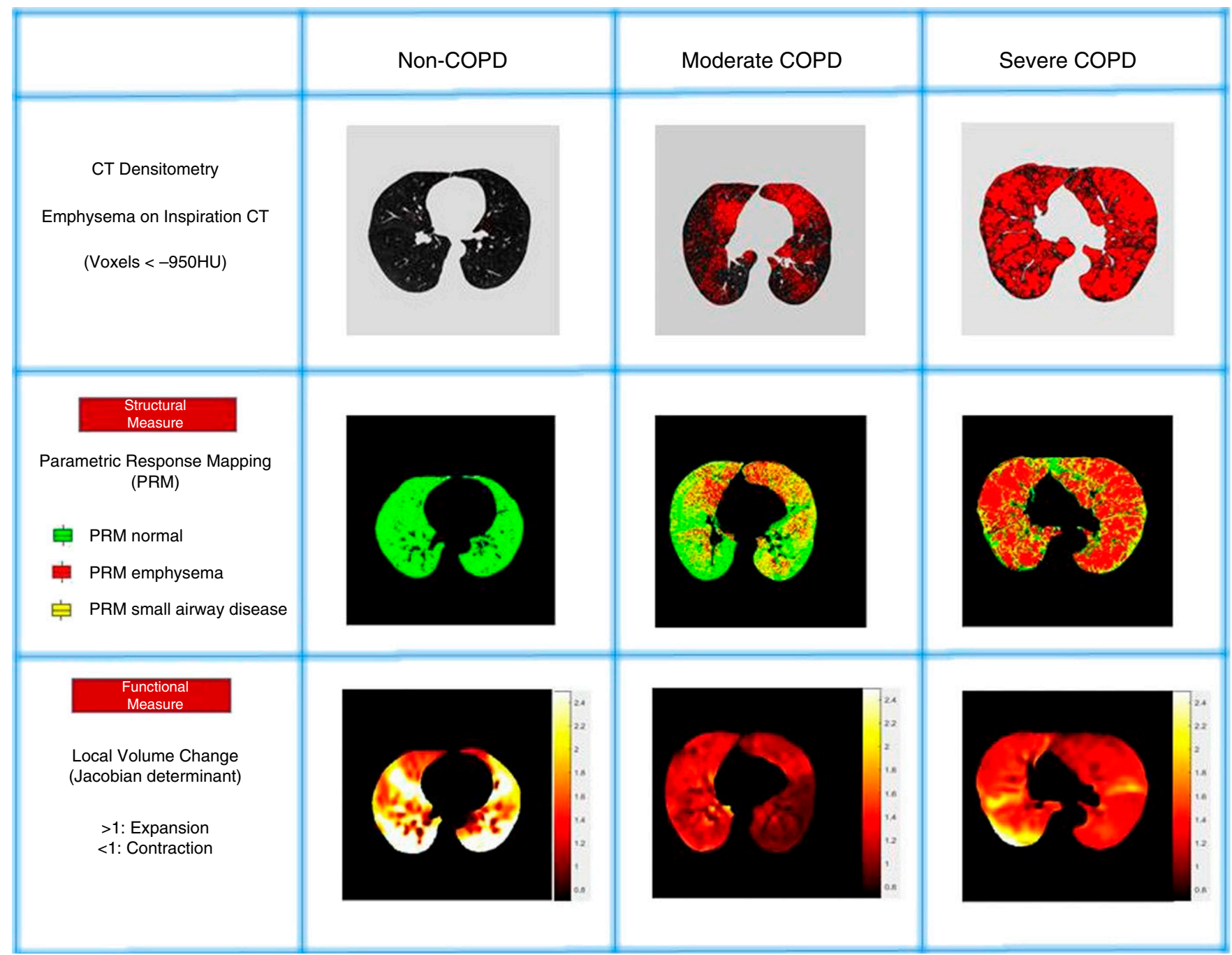

Figure 2. Differences in detection of structural lung disease using lung density measures (top panel) and after image registration with separation of emphysematous voxels and nonemphysematous gas trapping (middle panel). The bottom panel shows local volume change with respiration measured using the Jacobian determinant. Representative computed tomography (CT) scans from an individual without chronic obstructive pulmonary disease (COPD) and two individuals with moderate and severe COPD, respectively, are displayed. The figure demonstrates the differences in the three metrics for the same individuals using different tools for measuring parenchymal lung disease in COPD. PRM = parametric response mapping.

manifests as regions that do not show the expected increase in attenuation, reflecting either a reduced or absent change in gas volume during respiration. Dougherty and colleagues (53) subtracted matched inspiratory density maps from expiratory images and estimated the volume change in the areas of gas trapping. To improve spatial localization of volume change, Kim and associates (54) classified areas of the lung with density difference less than 50 $\mathrm{HU}$ as having gas trapping on paired inspiratory-expiratory scans (air trapping index), and assessed the degree of regional gas trapping in areas of normal, emphysematous, and hyperinflated lungs on baseline inspiratory scans. They found that this air trapping index correlated with $\mathrm{FEV}_{1} / \mathrm{FVC}(r=-0.74)$ and $\mathrm{FEV}_{1} \%$ predicted $(r=-0.73)$, with stronger correlations for the diseased regions of the lungs than normal lung regions. Unlike the gas trapping measures based on global density differences measured by the $\mathrm{E} / \mathrm{I}$ ratio, these metrics have the advantage of spatial localization, and hence can measure regional heterogeneity.

Specific volume change. Simple density difference metrics are confounded by the influence of the baseline inflation of the lung region of interest. Simon (55) described specific volume change $(\mathrm{sVol})$, which is the ratio of density change between inspiration and expiration over a tidal breath to the initial gas volume at inspiration. sVol has been validated against regional ventilation with xenon-CT in sheep, with a linear relationship between the two measures (56). Images acquired during tidal breathing can be substituted by images acquired at TLC and FRC, and the resultant $\mathrm{sVol}$ will reflect the regional volume change over that pressure and volume gradient. Coxson and colleagues (15) introduced another surrogate measure of regional ventilation, the specific regional gas volume $(\Delta \mathrm{SVg})$, by estimating the change in quantity of gas per gram of tissue between inspiration and expiration. They demonstrated a homogeneous distribution of $\Delta \mathrm{SVg}$ in healthy subjects and 
heterogeneous distribution in patients with COPD, with regions showing evident gas trapping associated with $\Delta \mathrm{SVg}$. All these metrics can be affected by the gravitational dependence of lung density, with dependent areas showing greater lung density. A comparison of the three metrics of regional ventilation (density difference, sVol, and $\Delta \mathrm{SVg}$ ) showed that $\Delta \mathrm{SVg}$ is least affected by gravity, and thus the heterogeneity seen in COPD is more directly related to the underlying pathology (57). These functional measures can detect additional heterogeneity over simple density maps. A possible explanation for the greater heterogeneity of functional measures in COPD than in normal subjects is difference in tissue compliance. An additional plausible reason is the presence of collateral channels and collateral airflow. In the healthy lung, collaterals have a minor effect on ventilation distribution, as they have up to 165-fold greater resistance to airflow than normal airways. However, in emphysematous lungs, collaterals can significantly impact regional ventilation as a result of decreased collateral resistance and increased airway resistance (58). Indeed, studies of regional ventilation have demonstrated an increase in local volume from inspiration to expiration in some areas, likely due to air entering from collaterals, and quantifying these areas has been proposed as a way of measuring collaterals (57).

\section{Jacobian measures of volume}

change. Lung deformation with respiration can be used to derive an alternative CT registration-based measure of regional ventilation termed the Jacobian determinant, a measure of local volume change from TLC to $\operatorname{FRC}(3,59)$. The deformation map created using registration represents pointwise expansion and contraction of lung areas, and has values ranging from 0 to infinity (Figure 3 ). A Jacobian determinant value greater than 1 indicates local expansion, less than 1 indicates local contraction, and a value of 1 represents neither local expansion nor contraction. Reinhardt and colleagues (60) estimated the Jacobian determinant-based local volume change, and showed good agreement with sVol measured on xenonCT. In patients with COPD, the Jacobian determinant has been shown to correlate strongly with $\mathrm{FEV}_{1} / \mathrm{FVC}(r=0.76)$ and $\mathrm{FEV}_{1} \%$ predicted $(r=0.80)(3,59)$, and is more strongly associated with airflow obstruction and respiratory quality of life than density measures of emphysema. Although there is strong correlation between CT emphysema and CT gas trapping with airflow obstruction, there is also substantial discordance in a number of patients with COPD (61). Values for the mean Jacobian determinant in life-long nonsmokers range from 1.3 to 2.5 , with an average of 2.0 (62). Bhatt and colleagues (3) showed that, in patients with substantial discordance between spirometric airflow obstruction and the degree of emphysema visualized on CT, the mean Jacobian determinant considerably improved prediction of spirometric airflow obstruction and also concordance between CT and spirometry measures of disease. Bodduluri and associates (63) showed that the mean Jacobian determinant for the entire lungs is independently associated with the body mass index, airflow obstruction, dyspnea, and exercise capacity index, (BODE) and thus offers additional prognostic information over that provided by traditional CT measures of structural disease (64). On follow-up, the mean Jacobian determinant approached statistical significance for predicting mortality (adjusted hazard ratio $=4.26 ; 95 \%$

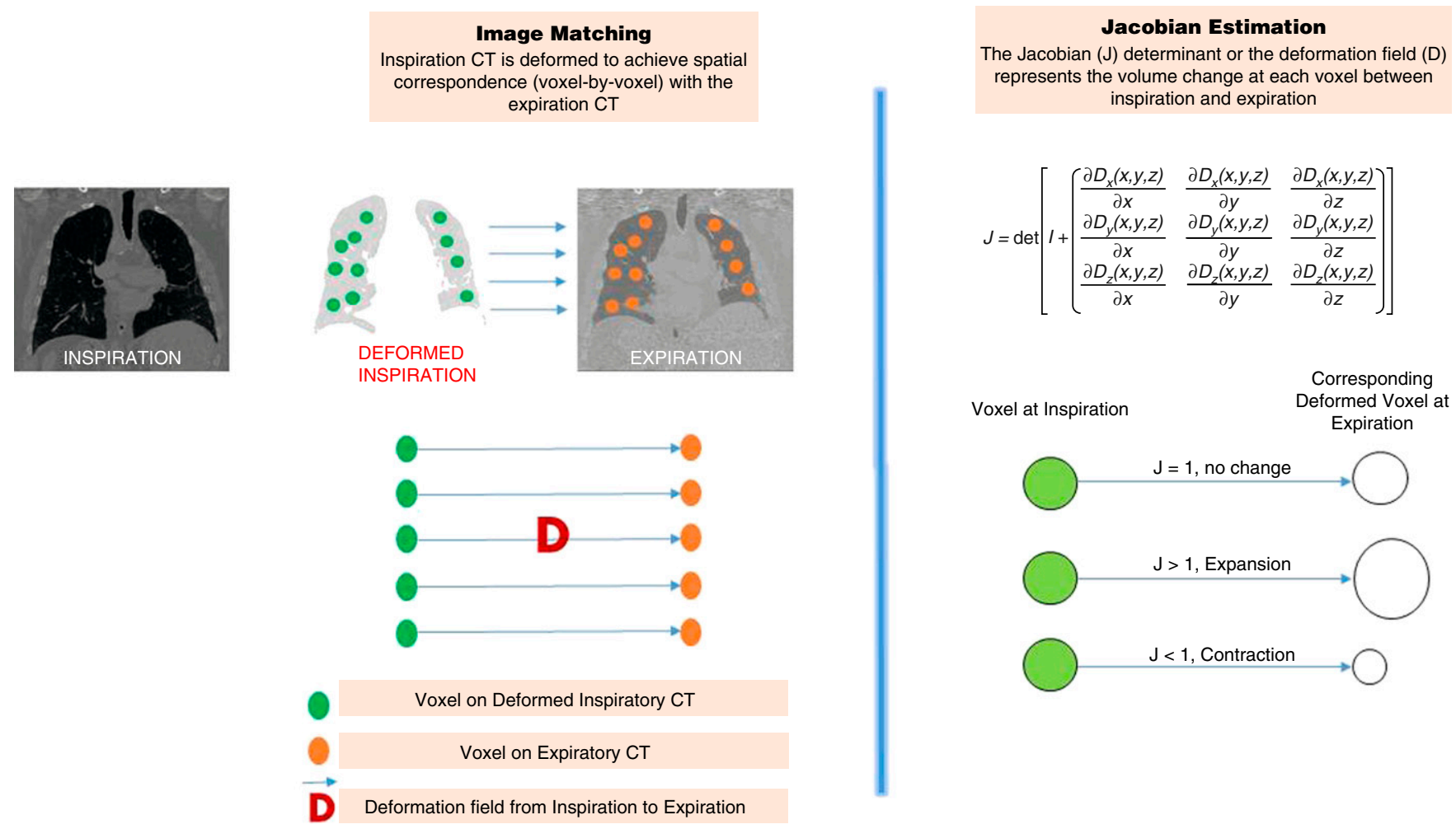

Figure 3. Image matching and derivation of the Jacobian determinant. CT= computed tomography, $\mathrm{D}=$ deformation field; $\mathrm{J}=\mathrm{Jacobian}$. 
confidence interval $=0.93-19.23 ; P=0.064$ ) (63). Bhatt and associates (65) used Jacobian metrics to show that normal voxels within $2 \mathrm{~mm}$ distance of emphysematous areas are mechanically influenced by the emphysematous areas, and this "mechanically affected lung" can be used to predict lung function decline.

\section{Lung Mechanics}

Although the density-based and Jacobianbased measures of regional ventilation quantify local volume change in the presence of disease, lung mechanics involve more than just volume change with respiration. Certain regions of the lung might undergo significant deformation, but no substantial volume change, especially when expansion in one axis is compensated by contraction along another axis. This is especially likely in the presence of heterogeneous adjacent lung. Based on this concept, Amelon and colleagues (66) developed the anisotropic deformation index, which captures the direction of lung deformation between inspiration and expiration using CT image registration. The lung deformation field can also be used to derive strain tensor, which is the ratio of the length of the deformed region of interest to the initial length of that region in the lung. Bodduluri and colleagues (59) combined the strain information, anisotropic deformation index, and Jacobian measure of volume change to create a lung biomechanical feature set. They compared the lung biomechanical feature set with CT density and texture-based measures of emphysema and gas trapping in identifying the presence and severity of COPD, and found that the biomechanical feature set was more accurate in COPD diagnosis and in severity classification. Estimation of mechanical forces can be useful in predicting disease progression over time (65).

\section{Practical Considerations and Limitations}

Although CT imaging can provide a rich source of information for the diagnosis and phenotyping of COPD, certain limitations exist. Considerable variability can occur between scans obtained at different time points, due to a number of potential sources of error, including scanner miscalibration, differences in reconstruction kernels used, and variability introduced by manufacturerdetermined reconstruction algorithms. Image comparison can also be affected by the lung volumes at which the images are acquired. Patient characteristics, such as obesity and active smoking, can influence lung density. Recently, reference equations were published for normative data for CT density parameters that can alleviate some of these sources of bias. Understandably, there remain concerns about radiation exposure with additional expiratory CT scans. Expiratory CT scans add an additional one-third to one-half of the inspiratory CT radiation exposure (25). Although the current radiation dose exposure for chest CT is approximately $7.0 \mathrm{mSv}$, multiple recent technological advances, including automatic exposure control, automatic tube potential selection, dynamic z-axis collimators, and beamshaping filters, as well as advances in iterative reconstruction algorithms and noise reduction methods, are likely to reduce radiation exposure two- to fourfold. It is anticipated that "submillisievert" scans will be possible in the near future, accounting for only one-third of the average background radiation exposure (67).

\section{Clinical Applications and Future Directions}

Although CT scans are not routinely obtained for patients with COPD in clinical practice, we believe CT imaging can be useful in a number of clinical scenarios. Patient selection for lung volume reduction procedures for severe emphysema, both surgical and bronchoscopic, is based on heterogeneity of emphysema on volumetric CT $(16,68,69)$. Fissure integrity can be assessed in an automated fashion, and at least $90 \%$ of fissure integrity is associated with better outcomes for bronchoscopic lung volume reduction (70). CT

densitometry can be used as a surrogate end point for disease progression in patients with $\alpha_{1}$-antitrypsin deficiency receiving augmentation therapy $(42,43)$. Densitybased and mechanics-based measures are also associated with respiratory morbidity, disease progression $(39,65,71)$, and mortality $(5,39,72)$, independent of lung function, thus providing additional information over and above that provided by spirometry alone. There is no definitive therapy to slow $\mathrm{FEV}_{1}$ decline. It is possible that the variability of $\mathrm{FEV}_{1}$ makes it less sensitive to changes induced by medications, and studies are underway to assess whether medications can influence the rate of progression of disease as measured by CT density (NCT00720226, available from www. clinicaltrials.gov) (73). A first step in targeting disease progression is detecting patients at risk, and CT has enabled identification of a number of biomarkers for disease progression, including assessment of functional small airways disease and mechanically affected lung $(39,65)$. Whether these CT biomarkers are modifiable remains to be investigated in future studies.

\section{Conclusions}

In summary, obtaining expiratory CT scans in conjunction with novel tools of image registration and parametric response mapping can add considerable anatomic and functional information in selected patients at risk for, or with, COPD. Measures of functional change in the lung parenchyma are likely to detect disease before becoming manifest on visual examination by a radiologist, and can also shed light on parenchymal heterogeneity that might aid patient selection for therapies, such as lung volume reduction. More research is needed to evaluate whether these emerging metrics can be used as intermediate end points for assessing response to pharmacotherapy.

Author disclosures are available with the text of this article at www.atsjournals.org.

\section{References}

1 Lynch DA, Newell JD. Quantitative imaging of COPD. J Thorac Imaging 2009;24:189-194.
2 Washko GR. Diagnostic imaging in COPD. Semin Respir Crit Care Med 2010;31:276-285.

3 Bhatt SP, Bodduluri S, Newell JD, Hoffman EA, Sieren JC, Han MK, et al.; COPDGene Investigators. CT-derived biomechanical metrics 
improve agreement between spirometry and emphysema. Acad Radiol 2016;23:1255-1263.

4 Lynch DA, Austin JH, Hogg JC, Grenier PA, Kauczor HU, Bankier AA, et al. CT-definable subtypes of chronic obstructive pulmonary disease: a statement of the Fleischner Society. Radiology 2015;277: 192-205.

5 Martinez CH, Chen YH, Westgate PM, Liu LX, Murray S, Curtis JL, et al.; COPDGene Investigators. Relationship between quantitative CT metrics and health status and BODE in chronic obstructive pulmonary disease. Thorax 2012;67:399-406.

6 Mishima M. CT imaging of COPD-importance of phenotyping (emphysema dominant and airway disorder dominant) [in Japanese]. Nihon Rinsho 2007;65:648-654.

7 Dirksen A. Is CT a new research tool for COPD? Clin Respir J 2008;2:76-83.

8 Hoffman EA, Lynch DA, Barr RG, van Beek EJ, Parraga G; IWPFI Investigators. Pulmonary CT and MRI phenotypes that help explain chronic pulmonary obstruction disease pathophysiology and outcomes. J Magn Reson Imaging 2016;43:544-557.

9 Gould GA, Redpath AT, Ryan M, Warren PM, Best JJ, Flenley DC, et al. Lung CT density correlates with measurements of airflow limitation and the diffusing capacity. Eur Respir J 1991;4:141-146.

10 Gould GA, MacNee W, McLean A, Warren PM, Redpath A, Best JJ, et al. CT measurements of lung density in life can quantitate distal airspace enlargement-an essential defining feature of human emphysema. Am Rev Respir Dis 1988;137:380-392.

11 Müller NL, Staples CA, Miller RR, Abboud RT. "Density mask": an objective method to quantitate emphysema using computed tomography. Chest 1988;94:782-787.

12 Madani A, De Maertelaer V, Zanen J, Gevenois PA. Pulmonary emphysema: radiation dose and section thickness at multidetecto CT quantification-comparison with macroscopic and microscopic morphometry. Radiology 2007;243:250-257.

13 Bankier AA, De Maertelaer V, Keyzer C, Gevenois PA. Pulmonary emphysema: subjective visual grading versus objective quantification with macroscopic morphometry and thin-section CT densitometry. Radiology 1999;211:851-858.

14 Gevenois PA, de Maertelaer V, De Vuyst P, Zanen J, Yernault J-C. Comparison of computed density and macroscopic morphometry in pulmonary emphysema. Am J Respir Crit Care Med 1995;152:653-657.

15 Coxson HO, Mayo JR, Behzad H, Moore BJ, Verburgt LM, Staples CA, et al. Measurement of lung expansion with computed tomography and comparison with quantitative histology. J Appl Physiol (1985) 1995;79:1525-1530.

16 Klooster K, ten Hacken NH, Hartman JE, Kerstjens HA, van Rikxoort EM, Slebos DJ. Endobronchial valves for emphysema without interlobar collateral ventilation. N Engl J Med 2015;373:2325-2335.

17 Cho MH, Castaldi PJ, Hersh CP, Hobbs BD, Barr RG, Tal-Singer R, et al.; NETT Genetics, ECLIPSE, and COPDGene Investigators. A genome-wide association study of emphysema and airway quantitative imaging phenotypes. Am J Respir Crit Care Med 2015; 192:559-569.

18 Lamers RJ, Thelissen GR, Kessels AG, Wouters EF, van Engelshoven JM. Chronic obstructive pulmonary disease: evaluation with spirometrically controlled CT lung densitometry. Radiology 1994;193:109-113.

19 Foreman MG, Zhang L, Murphy J, Hansel NN, Make B, Hokanson JE, et al.; COPDGene Investigators. Early-onset chronic obstructive pulmonary disease is associated with female sex, maternal factors, and African American race in the COPDGene Study. Am J Respir Crit Care Med 2011;184:414-420.

20 Hersh CP, Washko GR, Estépar RSJ, Lutz S, Friedman PJ, Han MK, et al.; COPDGene Investigators. Paired inspiratory-expiratory chest CT scans to assess for small airways disease in COPD. Respir Res 2013;14:42.

21 Matsuoka S, Kurihara Y, Yagihashi K, Hoshino M, Watanabe N, Nakajima Y. Quantitative assessment of air trapping in chronic obstructive pulmonary disease using inspiratory and expiratory volumetric MDCT. AJR Am J Roentgenol 2008;190:762-769.

22 Kim V, Davey A, Comellas AP, Han MK, Washko G, Martinez CH, et al.; COPDGene Investigators. Clinical and computed tomographic predictors of chronic bronchitis in COPD: a cross sectional analysis of the COPDGene study. Respir Res 2014;15:52.
23 Grydeland TB, Dirksen A, Coxson HO, Pillai SG, Sharma S, Eide GE, et al. Quantitative computed tomography: emphysema and airway wall thickness by sex, age and smoking. Eur Respir J 2009;34: 858-865.

24 Mair G, Maclay J, Miller JJ, McAllister D, Connell M, Murchison JT, et al. Airway dimensions in COPD: relationships with clinical variables. Respir Med 2010;104:1683-1690.

25 Sieren JP, Newell JD Jr, Barr RG, Bleecker ER, Burnette N, Carretta EE, et al.; SPIROMICS Research Group. SPIROMICS protocol for multicenter quantitative computed tomography to phenotype the lungs. Am J Respir Crit Care Med 2016;194:794-806.

26 Mohamed Hoesein FA, de Jong PA, Lammers JW, Mali WP, Schmidt $\mathrm{M}$, de Koning $\mathrm{HJ}$, et al. Airway wall thickness associated with forced expiratory volume in 1 second decline and development of airflow limitation. Eur Respir J 2015;45:644-651.

27 Mohamed Hoesein FA, de Jong PA, Lammers JW, Mali WP, Mets OM, Schmidt M, et al. Contribution of CT quantified emphysema, air trapping and airway wall thickness on pulmonary function in male smokers with and without COPD. COPD 2014;11:503-509.

28 Kim V, Desai P, Newell JD, Make BJ, Washko GR, Silverman EK, et al.; COPDGene Investigators. Airway wall thickness is increased in COPD patients with bronchodilator responsiveness. Respir Res 2014;15:84.

29 Bhatt SP, Wells JM, Kim V, Criner GJ, Hersh CP, Hardin M, et al.; COPDGene Investigators. Radiological correlates and clinical implications of the paradoxical lung function response to $\beta_{2}$ agonists: an observational study. Lancet Respir Med 2014;2: 911-918.

30 Gietema HA, Edwards LD, Coxson HO, Bakke PS; ECLIPSE Investigators Impact of emphysema and airway wall thickness on quality of life in smoking-related COPD. Respir Med 2013;107:1201-1209.

31 Woodruff PG, Barr RG, Bleecker E, Christenson SA, Couper D, Curtis JL et al.; SPIROMICS Research Group. Clinical significance of symptoms in smokers with preserved pulmonary function. N Engl J Med 2016; 374:1811-1821.

32 Smith BM, Hoffman EA, Rabinowitz D, Bleecker E, Christenson S Couper D, et al.; The Multi-Ethnic Study of Atherosclerosis (MESA) COPD Study and the Subpopulations and Intermediate Outcomes in COPD Study (SPIROMICS). Comparison of spatially matched airways reveals thinner airway walls in COPD. Thorax 2014;69: 987-996.

33 Washko GR, Diaz AA, Kim V, Barr RG, Dransfield MT, Schroeder J, et al. Computed tomographic measures of airway morphology in smokers and never-smoking normals. J Appl Physiol 1985;2014: 668-673.

34 Washko GR, Dransfield MT, Estepar RS, Diaz A, Matsuoka S, Yamashiro T, et al. Airway wall attenuation: a biomarker of airway disease in subjects with COPD. J Appl Physiol 1985;2009:185-191.

35 Bhatt SP, Terry NL, Nath H, Zach JA, Tschirren J, Bolding MS, et al.; Genetic Epidemiology of COPD (COPDGene) Investigators. Association between expiratory central airway collapse and respiratory outcomes among smokers. JAMA 2016;315:498-505.

36 Murphy K, van Ginneken B, Reinhardt JM, Kabus S, Ding K, Deng X, et al. Evaluation of registration methods on thoracic CT: the EMPIRE10 challenge. IEEE Trans Med Imaging 2011;30:1901-1920.

37 Galbán CJ, Han MK, Boes JL, Chughtai KA, Meyer CR, Johnson TD, et al. Computed tomography-based biomarker provides unique signature for diagnosis of COPD phenotypes and disease progression. Nat Med 2012;18:1711-1715.

38 McDonough JE, Yuan R, Suzuki M, Seyednejad N, Elliott WM, Sanchez $\mathrm{PG}$, et al. Small-airway obstruction and emphysema in chronic obstructive pulmonary disease. N Engl J Med 2011;365:1567-1575.

39 Bhatt SP, Soler X, Wang X, Murray S, Anzueto AR, Beaty TH, et al.; COPDGene Investigators. Association between functional small airways disease and $\mathrm{FEV}_{1}$ decline in chronic obstructive pulmonary disease. Am J Respir Crit Care Med 2016;194:178-184.

40 Boes JL, Hoff BA, Bule M, Johnson TD, Rehemtulla A, Chamberlain R, et al. Parametric response mapping monitors temporal changes on lung CT scans in the subpopulations and intermediate outcome measures in COPD Study (SPIROMICS). Acad Radiol 2015;22: 186-194. 
41 Parr DG, Sevenoaks M, Deng C, Stoel BC, Stockley RA. Detection of emphysema progression in alpha 1-antitrypsin deficiency using CT densitometry; methodological advances. Respir Res 2008;9:21.

42 Stockley RA, Parr DG, Piitulainen E, Stolk J, Stoel BC, Dirksen A. Therapeutic efficacy of $\alpha-1$ antitrypsin augmentation therapy on the loss of lung tissue: an integrated analysis of 2 randomised clinical trials using computed tomography densitometry. Respir Res 2010;11:136.

43 Chapman KR, Burdon JG, Piitulainen E, Sandhaus RA, Seersholm N, Stocks JM, et al.; RAPID Trial Study Group. Intravenous augmentation treatment and lung density in severe $\alpha 1$ antitrypsin deficiency (RAPID): a randomised, double-blind, placebo-controlled trial. Lancet 2015;386:360-368.

44 Washko GR, Kinney GL, Ross JC, San José Estépar R, Han MK, Dransfield MT, et al.; COPDGene Investigators. Lung mass in smokers. Acad Radiol 2017;24:386-392.

45 Gorbunova V, Lo P, Ashraf H, Dirksen A, Nielsen M, de Bruijne M. Weight preserving image registration for monitoring disease progression in lung CT. Med Image Comput Comput Assist Interv 2018;11(Pt 2): 863-870.

46 Gorbunova V, Sporring J, Lo P, Loeve M, Tiddens HA, Nielsen M, et al. Mass preserving image registration for lung CT. Med Image Anal 2012;16:786-795.

47 Gorbunova V, Jacobs SS, Lo P, Dirksen A, Nielsen M, Bab-Hadiashar $\mathrm{A}$, et al. Early detection of emphysema progression. Med Image Comput Comput Assist Interv 2010;13(pt 2):193-200.

48 Staring M, Bakker ME, Stolk J, Shamonin DP, Reiber JH, Stoel BC Towards local progression estimation of pulmonary emphysema using CT. Med Phys 2014;41:021905.

49 Choi S, Hoffman EA, Wenzel SE, Castro M, Lin CL. Improved CT-based estimate of pulmonary gas trapping accounting for scanner and lung-volume variations in a multicenter asthmatic study. $J$ Appl Physiol 1985;2014:593-603.

50 Suki B, Ito S, Stamenovic D, Lutchen KR, Ingenito EP. Biomechanics of the lung parenchyma: critical roles of collagen and mechanical forces. J Appl Physiol 1985;2005:1892-1899.

51 Pike D, Kirby M, Guo F, McCormack DG, Parraga G. Ventilation heterogeneity in ex-smokers without airflow limitation. Acad Radiol 2015;22:1068-1078.

52 Regan EA, Lynch DA, Curran-Everett D, Curtis JL, Austin JH, Grenier PA, et al.; Genetic Epidemiology of COPD (COPDGene) Investigators. Clinical and radiologic disease in smokers with normal spirometry. JAMA Intern Med 2015;175:1539-1549.

53 Dougherty L, Torigian DA, Affusso JD, Asmuth JC, Gefter WB. Use of an optical flow method for the analysis of serial CT lung images. Acad Radiol 2006;13:14-23.

54 Kim EY, Seo JB, Lee HJ, Kim N, Lee E, Lee SM, et al. Detailed analysis of the density change on chest CT of COPD using non-rigid registration of inspiration/expiration CT scans. Eur Radiol 2015;25: 541-549.

55 Simon BA. Regional ventilation and lung mechanics using X-ray CT. Acad Radiol 2005;12:1414-1422.

56 Fuld MK, Easley RB, Saba OI, Chon D, Reinhardt JM, Hoffman EA, et al. CT-measured regional specific volume change reflects regional ventilation in supine sheep. J Appl Physiol (1985) 2008;104: $1177-1184$

57 Aliverti A, Pennati F, Salito C, Woods JC. Regional lung function and heterogeneity of specific gas volume in healthy and emphysematous subjects. Eur Respir J 2013;41:1179-1188.

58 Hogg JC, Macklem PT, Thurlbeck WM. The resistance of collateral channels in excised human lungs. J Clin Invest 1969;48:421-431.
59 Bodduluri S, Newell JD Jr, Hoffman EA, Reinhardt JM. Registrationbased lung mechanical analysis of chronic obstructive pulmonary disease (COPD) using a supervised machine learning framework. Acad Radiol 2013;20:527-536.

60 Reinhardt JM, Ding K, Cao K, Christensen GE, Hoffman EA, Bodas SV. Registration-based estimates of local lung tissue expansion compared to xenon CT measures of specific ventilation. Med Image Anal 2008;12:752-763.

61 Bhatt SP, Sieren JC, Dransfield MT, Washko GR, Newell JD Jr, Stinson DS, et al.; COPDGene Investigators. Comparison of spirometric thresholds in diagnosing smoking-related airflow obstruction. Thorax 2014;69:409-414.

62 Bodduluri S. Analysis of chronic obstructive pulmonary disease (COPD) using CT images. 2012 [accessed 2017 Jul 22]. Available from: http:// ir.uiowa.edu/etd/2441

63 Bodduluri S, Bhatt SP, Hoffman EA, Newell JD Jr, Martinez CH, Dransfield MT, et al.; COPDGene Investigators. Biomechanical CT metrics are associated with patient outcomes in COPD. Thorax 2017; 72:409-414.

64 Celli BR, Cote CG, Marin JM, Casanova C, Montes de Oca M, Mendez RA, et al. The body-mass index, airflow obstruction, dyspnea, and exercise capacity index in chronic obstructive pulmonary disease. $N$ Engl J Med 2004;350:1005-1012.

65 Bhatt SP, Bodduluri S, Hoffman EA, Newell JD Jr, Sieren JC, Dransfield MT, et al.; COPDGene Investigators. Computed tomography measure of lung at risk and lung function decline in chronic obstructive pulmonary disease. Am J Respir Crit Care Med 2017;196: 569-576.

66 Amelon R, Cao K, Ding K, Christensen GE, Reinhardt JM, Raghavan $\mathrm{ML}$. Three-dimensional characterization of regional lung deformation. J Biomech 2011;44:2489-2495.

67 McCollough $\mathrm{CH}$, Chen GH, Kalender W, Leng S, Samei E, Taguchi K, et al. Achieving routine submillisievert CT scanning: report from the summit on management of radiation dose in CT. Radiology 2012; 264:567-580.

68 Fishman A, Martinez F, Naunheim K, Piantadosi S, Wise R, Ries A, et al.; National Emphysema Treatment Trial Research Group. A randomized trial comparing lung-volume-reduction surgery with medical therapy for severe emphysema. N Engl J Med 2003;348: 2059-2073.

69 Sciurba FC, Ernst A, Herth FJ, Strange C, Criner GJ, Marquette CH, et al.; VENT Study Research Group. A randomized study of endobronchial valves for advanced emphysema. N Engl J Med 2010; 363:1233-1244.

70 Schuhmann M, Raffy P, Yin Y, Gompelmann D, Oguz I, Eberhardt R, et al. Computed tomography predictors of response to endobronchial valve lung reduction treatment: comparison with Chartis. Am J Respir Crit Care Med 2015;191:767-774

71 Vestbo J, Edwards LD, Scanlon PD, Yates JC, Agusti A, Bakke P, et al.; ECLIPSE Investigators. Changes in forced expiratory volume in 1 second over time in COPD. N Engl J Med 2011;365:1184-1192.

72 Oelsner EC, Hoffman EA, Folsom AR, Carr JJ, Enright PL, Kawut SM, et al. Association between emphysema-like lung on cardiac computed tomography and mortality in persons without airflow obstruction: a cohort study. Ann Intern Med 2014;161:863-873.

73 Parikh MA, Aaron CP, Hoffman EA, Schwartz JE, Madrigano J, Austin JHM, et al.; The Multi-Ethnic Study of Atherosclerosis Lung Study. Angiotensinconverting inhibitors and angiotensin ii receptor blockers and longitudinal change in percent emphysema on computed tomography. Ann Am Thorac Soc 2017:14:649-658. 